\title{
Characterization of a common progenitor pool of the epicardium and myocardium
}

\author{
Richard C. V. Tyser'*, Ximena Ibarra-Soria ${ }^{2 *}$, Katie McDole ${ }^{3}$, Satish A. Jayaram¹, Jonathan Godwin', \\ Teun A. H. van den Brand ${ }^{4}$, Antonio M. A. Miranda ${ }^{1} \uparrow$, Antonio Scialdone ${ }^{5,6,7}$, Philipp J. Keller ${ }^{3}$, \\ John C. Marioni ${ }^{2,4,8}$ *, Shankar Srinivas ${ }^{1}$.
}

1Department of Physiology, Anatomy and Genetics, South Parks Road, University of Oxford, Oxford OX1 3QX, UK. ${ }^{2}$ Cancer Research UK Cambridge Institute, University of Cambridge, Li Ka Shing Centre, Robinson Way, Cambridge CB2 ORE, UK. ${ }^{3}$ Howard Hughes Medical Institute, Janelia Research Campus, 19700 Helix Drive, Ashburn, VA 20147, USA. ${ }^{4}$ European Molecular Biology Laboratory, European Bioinformatics Institute, Wellcome Genome Campus, Cambridge CB10 1SD, UK. ${ }^{5}$ nstitute of Epigenetics and Stem Cells, Helmholtz Zentrum München, D-81377 München, Germany. ${ }^{6}$ Institute of Functional Epigenetics, Helmholtz Zentrum München, D-85764 Neuherberg, Germany. ${ }^{7}$ Institute of Computational Biology, Helmholtz Zentrum München, D-85764 Neuherberg, Germany. ${ }^{8}$ Wellcome Sanger Institute, Wellcome Genome Campus, Cambridge CB10 1SA, UK.

*These authors contributed equally to this work.

†Present address: National Heart and Lung Institute, Imperial Centre for Translational and Experimental Medicine, Imperial College London, London W12 0NN, UK. ‡Corresponding author. Email: john.marioni@cruk.cam.ac.uk (J.C.M.); shankar.srinivas@dpag.ox.ac.uk (S.S.)

The mammalian heart is derived from multiple cell lineages; however, our understanding of when and how the diverse cardiac cell types arise is limited. We mapped the origin of the embryonic mouse heart at singlecell resolution using a combination of transcriptomic, imaging, and genetic lineage labeling approaches. This provided a transcriptional and anatomic definition of cardiac progenitor types. Furthermore, it revealed a cardiac progenitor pool that is anatomically and transcriptionally distinct from currently known cardiac progenitors. Besides contributing to cardiomyocytes, these cells also represent the earliest progenitor of the epicardium, a source of trophic factors and cells during cardiac development and injury. This study provides detailed insights into the formation of early cardiac cell types, with particular relevance to the development of cell-based cardiac regenerative therapies.

The heart is formed from several lineages, which give rise to the various cardiac cell types. The earliest mesodermal progenitors of cardiomyocytes emerge from the primitive streak, starting at approximately embryonic day (E)6.5 in the mouse (1-3). These cells migrate laterally across the egg cylinder to occupy an anterior position, initially forming the cardiac crescent before subsequently forming the linear heart tube (LHT) (4) (Fig. 1A). There are at least two distinct types of mesodermal cardiac progenitors, the first and second heart fields, defined broadly on the basis of marker genes expressed in different but overlapping anatomical locations (5). FHF cells occupy a craniolateral region and, together with early cardiomyocytes, form the cardiac crescent. The SHF represents a wider domain, dorsal and medial to the cardiac crescent. FHF cells show limited proliferation, instead rapidly differentiating into cardiomyocytes in order to lay down a primitive contractile apparatus (6) (Fig. 1A). In contrast, SHF cells are more proliferative, contributing to the growth of the heart before the cells differentiate. Given the overlap in expression of FHF and SHF markers, particularly with SHF cells upregulating FHF markers as they differentiate into cardiomyocytes, there is some debate about whether FHF and SHF correspond to fundamentally distinct cell types, or whether they represent different states along a shared differentiation trajectory from a common progenitor toward cardiomyocytes.

Retrospective clonal analyses support the idea that there are two separate lineages that contribute to the cardiomyocytes of the heart. The "first lineage" contributes primarily to the atria and left ventricles, whereas the "second lineage" contributes primarily to the atria, right ventricle, and outflow tract $(5,7)$. These two lineages are assumed to correspond to the FHF and SHF, respectively. However, it is unclear if the difference in fate of these two lineages is because of fundamental differences in their potential, or due only to differences in their embryonic location during early cardiac morphogenesis.

Cells from outside the traditional heart fields also contribute to the heart. Notably, the proepicardium gives rise to the epicardium, the outer layer of cells of the vertebrate heart. The epicardium generates several cardiac cell types including vascular smooth muscle cells and fibroblasts, in addition to providing important paracrine signals. It has attracted considerable attention as a possible source of cells and trophic factors in the context of repair after injury $(8,9)$. The proepicardium is initially situated at the inflow end of the looping heart tube at approximately E9.0, but its embryonic origin remains unclear (10-12). 
Single cell transcriptomics offers a powerful approach to characterize the various cell types of the embryonic heart and generate hypotheses about their lineal origins and fates. Several recent studies have used this approach to characterize cardiogenesis, focusing on subpopulations of cells isolated using predefined genes (13-16). Here, we perform a spatially resolved transcriptomic analysis of a microdissected region of the anterior embryo, collecting cells from the region corresponding to the embryonic heart over the time-course of cardiac crescent formation and LHT development.

\section{Single-cell analysis of heart formation}

We sampled cells, using manual microdissection to isolate the anterior cardiac region of mouse embryos, from early crescent to linear heart tube stages (Fig. 1, A and B; fig. S1A; and movie S1). Our collection was highly time-resolved, covering six defined stages spanning approximately 12 hours of development (roughly E7.75 to E8.25; fig. S1A) (17). Following quality control, the transcriptomes of 3105 cells were retained for further analysis (fig. S2A; Methods). After identifying genes with highly variable expression, we performed unsupervised clustering to determine the different cell types within our dataset. This revealed a total of 12 distinct clusters (Fig. 1, C and D), representing mesodermal cell types [including cardiac related (Me3-8) that represented a majority (66.1\%) of the cells, endothelial (Me2) and blood (Me1)], overlying endoderm [including definitive (En1) and yolk sac (En2)] and adjacent ectoderm [surface/amnion (Ec1) and neurectoderm (Ec2)] (18-20). We experimentally validated cluster identity using whole mount immunofluorescence for cluster-specific combinations of marker genes and single-cell resolution imaging (Fig. 1, E and F; figs. S3 and S4; and movie S2). As a user-friendly community resource, we have created a web-interface to interrogate these data, accessible at https://marionilab.cruk.cam.ac.uk/heartAtlas/.

Me3 represented the most differentiated cardiomyocytes within our dataset, showing elevated expression of contractile related genes Myh6, Actn2 and Ttn (Fig. 1D). Me3 started to emerge between stages 0 to 1 and increased over time (fig. $\mathrm{S} 1 \mathrm{~B})$, consistent with the formation of cardiomyocytes and onset of contractile activity at stage 1 (6). Me3 also showed reduced proliferative capacity in comparison to other clusters, with a higher proportion of cells in G1 (Fig. 1G and fig. S1C).

Me4 and Me6 were characterized by strong expression of cardiac progenitor markers, but intermediate to low levels of contractile genes (Fig. 1D) and therefore represented differentiating cardiac progenitors. Me5, Me7, and Me8 represented the least differentiated populations based on the low expression of contractile and cardiac physiology related genes (Fig. 1D).

\section{Transcriptional and spatial identification of cardiac progenitor subtypes}

To characterize these cardiac related clusters (Me3 to Me8) in more detail, we analyzed the expression of common FHF and SHF markers (Fig. $1 \mathrm{H}$ ). The cardiac progenitor marker Nkx2-5 was expressed in all these clusters except Me5 and Me8. Me4 had the highest combined expression of canonical FHF markers such as Tbx5, Nkx2-5, Hcn4 and Sfrp5, although some of these were also found in Me3, Me5 and to a lesser extent, Me7. Me6 and Me7 showed a distinct SHF-like signature (Fig. $1 \mathrm{H}$ ), although again, some SHF markers were also seen in Me3 and Me5.

The FHF and SHF are defined not only based on markers but also on their anatomical location. Hence, we next confirmed our assignment of clusters to a FHF or SHF identity by generating a spatially resolved scRNA-seq reference dataset from four anatomically defined regions of mesoderm in stage 3 crescent embryos ( E8.0). We manually microdissected and sequenced cells from the ventral mesoderm containing the cardiac crescent (VM), abutting dorsal mesoderm (DM), cranial mesoderm (CrM), and paraxial mesoderm (PM) (Fig. 2A). We generated an average of 70 high-quality singlecell transcriptomes for each of these reference regions, which together clustered into five different subpopulations (clusters 1 to 5) that were dispersed to varying degrees across the four regions (Fig. 2B and fig. S5A). Cluster 1 expressed the cranial mesoderm marker Cyp26c1 (21) (Fig. 2C) and was almost exclusively derived from this reference region. Cluster 2 represented paraxial mesoderm (Tcf15 and MeoxI) $(22,23)$ and was composed of cells from this region as well as from the dorsal mesoderm. Clusters 3, 4 and 5 expressed cardiac markers such as Ttn and Gata5 (24) and were made up predominantly of cells from the VM and DM (Fig. 2, B and C). VM cells could be categorized as more mature, differentiated cardiac cells (cluster 5), and less mature "cardiac-like" cells (clusters 3 and 4) (Fig. 2B and fig. S5A). Similarly, DM cells were split across "cardiac-like" and "PM-like" (clusters 3 and 2, respectively; Fig. 2B). Hoxa1, which marks cells from the DM, highlights the pronounced transcriptional heterogeneity within this region, being split across clusters 2 and 3 (Fig. 2, B and C).

To use these reference cells to guide spatial localization of the cells assigned to clusters Me3-8, we trained a random forest classifier using the combined information from the dissection labels and clustering analysis (Methods; Fig. 2B and fig. S5, A and B). Upon application of this classifier to the main dataset, the majority of cells in clusters Me3-6 were assigned to the VM (Fig. 2, D and E), which corresponds broadly to the cardiac crescent. Consistent with the differences in maturity observed between Me5 and Me3/4/6, the former matched "cardiac-like" VM cells while the latter mapped to the more mature cardiac VM (Fig. 2, D and E). The majority of the cells from Me7 mapped to "cardiac-like" DM reference 
cells whereas Me8 mapped predominantly to CrM but also PM-like DM (Fig. 2, D and E). In particular, although Me7 and Me8 were both characterized by high Tbx1 and Foxc2, they could be distinguished by expression of $N k x 2-5$ (high in Me7, low in Me8) and Fst (low in Me7, high in Me8) (Fig. 2, F and G; figs. S6 to S8; and movies S3 to S5 and S7). This allowed us to localize Me7 to areas of mesoderm medial and dorsal to the crescent, consistent with it representing cells from the SHF.

\section{Intermediate states in cardiomyocyte differentiation are spatially localized across development}

We next employed a diffusion map approach to infer differentiation trajectories between clusters Me3-7, excluding Me8 due to its CrM and PM-like identity. This analysis revealed two distinct trajectories, both leading to the differentiated cardiomyocytes of Me3 (Fig. 2H and fig. S9). One trajectory connected Me7 to Me3 via two distinct intermediates, Me6 and Me4. The second trajectory linked Me5 to Me3 via Me4 (Fig. $2 \mathrm{H}$ and fig. S9, A to C).

The trajectory linking Me5, Me4 and Me3 is consistent with a FHF-like differentiation trajectory. Although Me4 had a broad FHF transcriptional signature, Me5 did not fit with straightforward categorization as it expressed some FHF markers (Hand1 and Tbx5), but significantly lower levels of the canonical cardiac progenitor marker Nkx2-5 (Fig. 1, D and $\mathrm{H})$. Therefore, to further characterize these clusters, we determined their precise embryonic locations in $3 \mathrm{D}$ at different stages using the combinatorial expression of multiple markers (fig. S10) and single-cell resolution volume imaging.

Me5 cells are Hand1 and Snai1 positive but also Nkx2-5 negative. Using these and other markers such as Smarcd3 and $T b x 5$, we were able to locate Me5 at the rostral border of the cardiac crescent, as a discrete group of cells at the confluence of extraembryonic and splanchnic mesoderm, corresponding anatomically with the primitive septum transversum (25) (Fig. 3, A to C, and fig. S11). The splanchnic mesoderm here is a monolayer continuous with the extraembryonic mesoderm of the yolk-sac. Within this sheet, at stage 1 and earlier, Me5 was rostrally positioned with respect to the more caudal NKX2-5 and TITIN positive cardiac progenitors (Fig. 3B). Later, at stage 2, rostral folding causes this mesoderm to buckle, such that Me 5 is now sandwiched between the endoderm and the more dorsal layer of NKX2-5 positive cardiac progenitors, while maintaining its relative positions within the plane of the mesodermal sheet (Fig. 3, C and D). Using our scRNA-seq data we identified Mab21l2 as a marker of Me5 (Fig. 3, E and F; figs. S8B and S12; and movies S6 and S7). Expression of Mab21l2 confirmed the location of Me5 at the rostral border of the crescent prior to rostral folding, and between the endoderm and crescent during folding (Fig. 3E and fig. S12).
Me6, characterized by overlapping expression of $A s b 2$ and $T b x 1$, represented a small population of cells restricted to the medial-dorsal interface between $\mathrm{Me} 7$ and the cardiac crescent (Fig. 4, A and F; fig. S13; and movie S3), consistent with it representing a transitional state during the differentiation of SHF progenitors (Me7) into cardiomyocytes (Me3).

Me4, identified by expression of Vsnl1 and absence of $F s d 2$, localized at stage 1 to a narrow arc within the cardiac crescent, at the interface between Me5 and Me3 (Fig. 4, B, C, and F; figs. S14 and S15; and movies S8 to S10). At stage -1 , prior to formation of cardiomyocytes (Me3), Me4 formed the earliest arcs of the cardiac crescent, just caudal to Me5 (Fig. 4, D and F; figs. S16 and S17; and movie S11), consistent with it representing the FHF. The discrete anatomical locations of these transcriptional clusters highlights the spatially graded maturation of cardiomyocytes in this region. The Me4 state could be detected as late as the LHT stage (fig. S14C).

Our diffusion analysis indicated that $\mathrm{Me}$ ( $\mathrm{SHF}$ ) could contribute to Me3 (cardiomyocytes) via not only Me6, a transient transcriptional state specific to the SHF trajectory, but also via Me4, that captures the FHF transcriptional state. Consistent with this, we detected Me4 cells not only at the interface between Me5 and Me3 (above) but also at the interface of Me7 and Me3 (Fig. 4, E and F, and figs. S16C and S17, $\mathrm{C}$ and $\mathrm{D}$ ). In addition, Me3, defined by the expression of $F s d 2$, could be separated into two subgroups based on the presence or absence of the Me4 marker Vsnl1. Imaging revealed that the Vsnl1 positive cardiomyocytes of Me3 were located in the ventral crescent, adjacent to Me4, while the Vsnl1 negative cardiomyocytes were located more medial-dorsally, adjacent to $\mathrm{Me} 7$ and Me6 (Fig. 4C and fig. S14, D and E). Given the different anatomical locations and transcriptional profiles of SHF intermediates, the branched trajectory of SHF progenitors possibly reflects the early separation of anterior and posterior SHF.

The consensus FHF transcriptional signature and anatomical location of Me4 within the crescent indicate that it represents the FHF. In contrast, the $N k x 2-5$ negative Me 5 corresponds to progenitors of the FHF. Hand1 has been considered a FHF marker due to in situ hybridization using chromogenic detection that appears to label the cardiac crescent (26). However, our single-cell resolution imaging shows that HAND1 in fact marks Me5, which is distinct from the FHF and cardiac crescent (Fig. 3, A to C); due to its ventral position overlying the crescent, volume imaging is required to distinguish the two. Given the proximity of Me5 to the cardiac crescent, we named it the juxta-cardiac field (JCF).

\section{The JCF represents a cardiac progenitor population}

To determine if the JCF, similar to other cardiomyocyte progenitors, is derived from cells that expressed Mesp1, we used a Mesp1-Cre (3) driver line. This verified that the JCF arises 
from Mesp1 positive mesoderm (Fig. 5A). Similarly, using an Nkx2-5-Cre (26) driver line showed that JCF cells had never previously expressed $N k x 2-5$ (Fig. 5A).

Supporting a differentiation trajectory from the JCF to cardiomyocytes, the cardiac progenitor gene $N k x 2-5$ increased in expression along pseudotime as cells transitioned from JCF to Me3 (Fig. 5B). We therefore tracked JCF cells in embryos where expression of $N k x 2-5$ could be monitored using nuclear-eGFP fluorescence $(27,28)$ using single-cell resolution light-sheet microscopy (29). Confirming the diffusion map trajectory, we found that JCF cells activated expression of Nkx2-5:GFP as they moved toward the cardiac crescent during rostral folding (Fig. 5, $\mathrm{C}$ to $\mathrm{E}$, and two representative cells in movies S12 and S13).

The Me5 marker Mab21l2 is expressed rostral to the cardiac crescent and is down-regulated during cardiomyocyte differentiation (Fig. 5F). Prior to crescent formation, at early headfold stages (30), Mab21l2 was detected rostral to an Nkx 2-5-positive domain, at the boundary between embryonic and extraembryonic mesoderm (Fig. 5F, fig. S18B, and movie S14). At early pre-headfold stages, we could not detect Mab21l2, but found Nkx2-5 expression in the anterior mesoderm (fig. S18C). This suggests that the Me5 population forms after other cardiac progenitor populations.

Our transcriptomic data showed that Me8, Me4 and a small subset of Me5 expressed Tbx18 (Fig. 5G). A Tbx18 positive (and Nkx2-5, Isl1 double negative) population of caudallateral mesoderm can give rise to sinus venosus myocardium (31). To test whether the JCF and sinus venosus precursors might overlap, we assayed for $T b x 18$ and Mab21l2 coexpression by in situ hybridization chain reaction (Fig. 5G and fig. S19). In contrast to Mab21l2, Tbx18 was not detected until stage 1, with weak expression in two separate domains, a region of the lateral crescent and medial paraxial mesoderm, corresponding with Me8 (Fig. 5G). In contrast, Mab21l2, was expressed earlier, at early headfold stages, rostrally and in a significantly larger region covering both medial and lateral regions of mesoderm (fig. S19B). Tbx18 was never detected in the rostral-medial JCF, indicating that the JCF is a spatially and transcriptionally distinct progenitor population from the sinus venosus progenitors (Fig. 5G).

\section{Juxta-cardiac field progenitors contribute to cardiomy- ocytes and epicardium}

In order to test our diffusion trajectory results indicating the JCF gives rise to cardiomyocytes, we knocked CreERT2 into the Mab2l12 locus in a homozygous R26R-YFP reporter background (32). At E8.0, nuclear localized CreERT2 was found only in the juxta-cardiac field and was not detected in cardiomyocytes (fig. S20B).

To specifically label the JCF, we administered a single low dose of tamoxifen to pregnant dams at $6.5 \mathrm{dpc}$ then assessed the fate of labeled cells at several subsequent time points (Fig. $6 \mathrm{~A})$. This ensured that tamoxifen was available when Mab2l12 first begins to mark the JCF around E7.5 while minimizing the possibility of labeling cells outside the JCF, or at subsequent stages. This protocol allowed us to stringently assess the fate of the JCF but likely underrepresents the contribution of this progenitor pool due to the early, limiting, dose of tamoxifen.

We first tested specificity of labeling by looking in E8.0 embryos. Fewer than half of Mab2l12-CreERT2 transgenic embryos showed any labeled cells (19 out of 45 embryos) consistent with the limiting dose of tamoxifen. YFP positive cells could be found only in the JCF at early crescent stages and did not express markers of maturing FHF cells such as sarcomeric $\alpha$-actinin or cardiac Troponin T (cTnT) (Fig. 6B and fig. $\mathrm{S} 20$, B to D). On average, five YFP positive cells could be detected per embryo between stage 1 and LHT (fig. S20A), highlighting the low level of recombination. These data confirmed that the new Mab21l2-iCreERT2 transgenic line specifically targeted JCF cells and would allow us to investigate their fate.

We next analyzed the early looping heart tube at E8.5. Consistent with time-lapse results (Fig. 5D) this revealed that JCF cells contribute to cardiomyocytes within the linear heart tube, as verified by sarcomeric striations of alpha-Actinin in YFP positive cells (Fig. $6 \mathrm{C}$ and table S1). Additionally, the JCF was maintained throughout these stages as YFP positive noncardiomyocyte cells were still present in the JCF domain, which by this stage was positioned by rostral folding between the cardiac tube and endoderm (Fig. 6C).

JCF cells, in addition to forming cardiomyocytes, also contributed to the proepicardium at E9.5. Whole mount immunofluorescence for WT1, a proepicardium and epicardium marker, revealed colocalization with YFP cells (Fig. 6D and table S2). Consistent with this, at E10.5 we found YFP positive cells in both the epicardium and myocardium. The latter cells were found in the left ventricle, atrioventricular canal and atria, but never in the right ventricle or outflow tract (Fig. 6, $\mathrm{E}$ and F, and tables S3 and S4). The direct contribution of the JCF to myocardium, as opposed to via differentiation of later formed epicardial cells, is evident from the JCF derived myocardial cells observed already at E8.5, before the formation of the epicardium (Fig. 6C and tables S1 and S2). These data together establish the juxta-cardiac field as a progenitor pool of cardiomyocytes and the proepicardium.

Our spatially and temporally resolved single-cell anatomical and transcriptional analysis provides a multifaceted resource for understanding how the heart forms. It also provides a spatial and transcriptomic reference definition of the FHF, SHF, and intermediate states to cardiomyocytes. Given that the transcriptional state of a cell is labile, but scRNA-seq offers only a snapshot of its transcriptional state, one has to be cautious in using "marker" genes to make 
inferences regarding lineage (past history, often with the implicit assumption that a shared transcriptional state implies a shared history) and fate (the future descendants). This is particularly evident in Me4 that represents a FHF transcriptional state, but through which cells of two different origins (JCF or SHF) can transition in forming cardiomyocytes. It also highlights the importance of using independent approaches such as genetic fate mapping to determine the fate of cells.

Our analyses revealed a progenitor pool, the JCF, that can contribute to cardiomyocytes via a FHF transcriptional state, as well as to the proepicardium. The JCF is located at the rostral confluence of the embryonic and extraembryonic mesoderm, and may represent a subset of the forming septum transversum. Although the JCF can contribute to the FHF, it is unlikely to be the sole progenitor. It is currently unclear if the JCF is a pool of unipotent progenitors of cardiomyocytes and proepicardium, or contains bipotent cells capable of giving rise to both. Future clonal analyses are required to address this question. The significance of the JCF is supported by a previous study which showed that loss of function of the JCF marker Mab21l2 leads to early embryonic lethality due to a reduction in myocardium and proepicardium (33). Our work further indicates there are likely multiple populations contributing to the proepicardium, the JCF representing an early population, and the $T b x 18$ positive population of paraxial mesoderm (10-12), a later population.

By identifying the juxta-cardiac field, our work widens the cardiac progenitor region, identifies the earliest progenitors of the proepicardium and provides new insights into the lineal origins of cardiac cell types. This, combined with the detailed characterization of these cell types at the transcriptomic level, will contribute to a better understanding of the origin of congenital cardiac defects as well as provide basic insights for informing regenerative approaches to treating heart disease.

\section{Materials and methods summary}

All animal experiments complied with the UK Animals (Scientific Procedures) Act 1986, approved by the local Biological Services Ethical Review Process and were performed under UK Home Office project licenses PPL 30/3420 and PCB8EF1B4. Anterior embryonic regions were dissected manually, dissociated to single cells and processed to obtain single-cell transcriptomes. Single cell analyses, whole mount immunohistochemistry, in situ hybridization chain reaction, volume imaging, light-sheet live microscopy, 2C-HR-CRISPR based genetic engineering and genetic lineage labeling are described in detail in the supplementary methods.

\section{REFERENCES AND NOTES}

1. K. A. Lawson, Fate mapping the mouse embryo. Int. J. Dev. Biol. 43, 773-775 (1999). Medline

2. M. Parameswaran, P. P. L. Tam, Regionalisation of cell fate and morphogenetic movement of the mesoderm during mouse gastrulation. Dev. Genet. 17, 16-28 (1995). doi:10.1002/dvg.1020170104 Medline

3. Y. Saga, S. Miyagawa-Tomita, A. Takagi, S. Kitajima, Ji. Miyazaki, T. Inoue, MesP1 is expressed in the heart precursor cells and required for the formation of a single heart tube. Development 126, 3437-3447 (1999). Medline

4. S. M. Meilhac, M. E. Buckingham, The deployment of cell lineages that form the mammalian heart. Nat. Rev. Cardiol. 15, 705-724 (2018). doi:10.1038/s41569018-0086-9 Medline

5. F. Lescroart, S. Chabab, X. Lin, S. Rulands, C. Paulissen, A. Rodolosse, H. Auer, Y. Achouri, C. Dubois, A. Bondue, B. D. Simons, C. Blanpain, Early lineage restriction in temporally distinct populations of Mesp1 progenitors during mammalian heart development. Nat. Cell Biol. 16, 829-840 (2014). doi:10.1038/ncb3024 Medline

6. R. C. V. Tyser, A. M. A. Miranda, C. M. Chen, S. M. Davidson, S. Srinivas, P. R. Riley, Calcium handling precedes cardiac differentiation to initiate the first heartbeat. eLife 5, e17113 (2016). doi:10.7554/eLife.17113 Medline

7. S. M. Meilhac, M. Esner, R. G. Kelly, J. F. Nicolas, M. E. Buckingham, The clonal origin of myocardial cells in different regions of the embryonic mouse heart. Dev. Cell 6 685-698 (2004). doi:10.1016/S1534-5807(04)00133-9 Medline

8. J. Cao, K. D. Poss, The epicardium as a hub for heart regeneration. Nat. Rev. Cardiol. 15, 631-647 (2018). doi:10.1038/s41569-018-0046-4 Medline

9. F. C. Simões, P. R. Riley, The ontogeny, activation and function of the epicardium during heart development and regeneration. Development 145, dev155994 (2018). doi:10.1242/dev.155994 Medline

10. M. T. M. Mommersteeg, J. N. Domínguez, C. Wiese, J. Norden, C. de Gier-de Vries, J. B. E. Burch, A. Kispert, N. A. Brown, A. F. M. Moorman, V. M. Christoffels, The sinus venosus progenitors separate and diversify from the first and second heart fields early in development. Cardiovasc. Res. 87, 92-101 (2010) doi:10.1093/cvr/cvq033 Medline

11. J. Schlueter, T. Brand, Subpopulation of proepicardial cells is derived from the somatic mesoderm in the chick embryo. Circ. Res. 113, 1128-1137 (2013). doi:10.1161/CIRCRESAHA.113.301347 Medline

12. M. Bressan, G. Liu, T. Mikawa, Early mesodermal cues assign avian cardiac pacemaker fate potential in a tertiary heart field. Science 340, 744-748 (2013). doi:10.1126/science. 1232877 Medline

13. G. Li, A. Xu, S. Sim, J. R. Priest, X. Tian, T. Khan, T. Quertermous, B. Zhou, P. S. Tsao, S. R. Quake, S. M. Wu, Transcriptomic profiling maps anatomically patterned subpopulations among single embryonic cardiac cells. Dev. Cell 39, 491-507 (2016). doi:10.1016/i.devcel.2016.10.014 Medline

14. F. Lescroart, X. Wang, X. Lin, B. Swedlund, S. Gargouri, A. Sànchez-Dànes, V. Moignard, C. Dubois, C. Paulissen, S. Kinston, B. Göttgens, C. Blanpain, Defining the earliest step of cardiovascular lineage segregation by single-cell RNA-seq. Science 359, 1177-1181 (2018). doi:10.1126/science.aa04174 Medline

15. T. Y. de Soysa, S. S. Ranade, S. Okawa, S. Ravichandran, Y. Huang, H. T. Salunga, A. Schricker, A. Del Sol, C. A. Gifford, D. Srivastava, Single-cell analysis of cardiogenesis reveals basis for organ-level developmental defects. Nature $\mathbf{5 7 2}$, 120-124 (2019). doi:10.1038/s41586-019-1414-x Medline

16. H. Xiong, Y. Luo, Y. Yue, J. Zhang, S. Ai, X. Li, X. Wang, Y.-L. Zhang, Y. Wei, H.-H. Li, X. Hu, C. Li, A. He, Single-cell transcriptomics reveals chemotaxis-mediated intraorgan crosstalk during cardiogenesis. Circ. Res. 125, 398-410 (2019) doi:10.1161/CIRCRESAHA.119.315243 Medline

17. R. C. V. Tyser, S. Srinivas, The first heartbeat-origin of cardiac contractile activity Cold Spring Harbor Perspect. Biol. 12, a037135 (2020). doi:10.1101/cshperspect.a037135 Medline

18. S. L. Paige, K. Plonowska, A. Xu, S. M. Wu, Molecular regulation of cardiomyocyte differentiation. Circ. Res. 116, 341-353 (2015). doi:10.1161/CIRCRESAHA.116.302752 Medline

19. S. Nowotschin, M. Setty, Y.-Y. Kuo, V. Liu, V. Garg, R. Sharma, C. S. Simon, N. Saiz, R. Gardner, S. C. Boutet, D. M. Church, P. A. Hoodless, A.-K. Hadjantonakis, D. Pe'er, The emergent landscape of the mouse gut endoderm at single-cell resolution. Nature 569, 361-367 (2019). doi:10.1038/s41586-019-1127-1 Medline 
20. B. Pijuan-Sala, J. A. Griffiths, C. Guibentif, T. W. Hiscock, W. Jawaid, F. J. CaleroNieto, C. Mulas, X. Ibarra-Soria, R. C. V. Tyser, D. L. L. Ho, W. Reik, S. Srinivas, B. D. Simons, J. Nichols, J. C. Marioni, B. Göttgens, A single-cell molecular map of mouse gastrulation and early organogenesis. Nature 566, 490-495 (2019). doi:10.1038/s41586-019-0933-9 Medline

21. H. Wang, P. W. H. Holland, T. Takahashi, Gene profiling of head mesoderm in early zebrafish development: Insights into the evolution of cranial mesoderm. EvoDevo 10, 14 (2019). doi:10.1186/s13227-019-0128-3 Medline

22. R. Burgess, A. Rawls, D. Brown, A. Bradley, E. N. Olson, Requirement of the paraxis gene for somite formation and musculoskeletal patterning. Nature 384, 570-573 (1996). doi:10.1038/384570a0 Medline

23. A. F. Candia, J. Hu, J. Crosby, P. A. Lalley, D. Noden, J. H. Nadeau, C. V. Wright, Mox-1 and Mox-2 define a novel homeobox gene subfamily and are differentially expressed during early mesodermal patterning in mouse embryos. Development 116, 1123-1136 (1992). Medline

24. J. F. Reiter, J. Alexander, A. Rodaway, D. Yelon, R. Patient, N. Holder, D. Y. R. Stainier, Gata5 is required for the development of the heart and endoderm in zebrafish. Genes Dev. 13, 2983-2995 (1999). doi:10.1101/gad.13.22.2983 Medline

25. S. L. Dunwoodie, T. A. Rodriguez, R. S. P. Beddington, Msg1 and Mrg1, founding members of a gene family, show distinct patterns of gene expression during mouse embryogenesis. Mech. Dev. 72, 27-40 (1998). doi:10.1016/S09254773(98)00011-2 Medline

26. K. A. Moses, F. DeMayo, R. M. Braun, J. L. Reecy, R. J. Schwartz, Embryonic expression of an Nkx2-5/Cre gene using ROSA26 reporter mice. Genesis 31, 176180 (2001). doi:10.1002/gene.10022 Medline

27. E. G. Stanley, C. Biben, A. Elefanty, L. Barnett, F. Koentgen, L. Robb, R. P. Harvey, Efficient Cre-mediated deletion in cardiac progenitor cells conferred by a 3'UTRires-Cre allele of the homeobox gene Nkx2-5. Int. J. Dev. Biol. 46, 431-439 (2002). Medline

28. J. R. Prigge, J. A. Wiley, E. A. Talago, E. M. Young, L. L. Johns, J. A. Kundert, K. M. Sonsteng, W. P. Halford, M. R. Capecchi, E. E. Schmidt, Nuclear doublefluorescent reporter for in vivo and ex vivo analyses of biological transitions in mouse nuclei. Mamm. Genome 24, 389-399 (2013). doi:10.1007/s00335-0139469-8 Medline

29. K. McDole, L. Guignard, F. Amat, A. Berger, G. Malandain, L. A. Royer, S. C. Turaga, K. Branson, P. J. Keller, In toto imaging and reconstruction of post-implantation mouse development at the single-cell level. Cell 175, 859-876.e33 (2018). doi:10.1016/i.cell.2018.09.031 Medline

30. K. A. Lawson, V. Wilson, "A revised staging of mouse development before organogenesis" in Kaufman's Atlas of Mouse Development Supplement (Academic Press, 2016), pp. 51-64.

31. V. M. Christoffels, M. T. M. Mommersteeg, M.-0. Trowe, O. W. J. Prall, C. de Gierde Vries, A. T. Soufan, M. Bussen, K. Schuster-Gossler, R. P. Harvey, A. F. M. Moorman, A. Kispert, Formation of the venous pole of the heart from an Nkx2-5negative precursor population requires Tbx18. Circ. Res. 98, 1555-1563 (2006). doi:10.1161/01.RES.0000227571.84189.65 Medline

32. S. Srinivas, T. Watanabe, C.-S. Lin, C. M. William, Y. Tanabe, T. M. Jessell, F. Costantini, Cre reporter strains produced by targeted insertion of EYFP and ECFP into the ROSA26 locus. BMC Dev. Biol. 1, 4 (2001). doi:10.1186/1471-213X-1-4 Medline

33. Y. Saito, T. Kojima, N. Takahashi, Mab21/2 is essential for embryonic heart and liver development. PLOS ONE 7, e32991 (2012). doi:10.1371/journal.pone.0032991 Medline

34. S. Picelli, O. R. Faridani, Å. K. Björklund, G. Winberg, S. Sagasser, R. Sandberg, Fulllength RNA-seq from single cells using Smart-seq2. Nat. Protoc. 9, 171-181 (2014). doi:10.1038/nprot2014.006 Medline

35. T. D. Wu, S. Nacu, Fast and SNP-tolerant detection of complex variants and splicing in short reads. Bioinformatics 26, 873-881 (2010). doi:10.1093/bioinformatics/bta057 Medline

36. H. Li, B. Handsaker, A. Wysoker, T. Fennell, J. Ruan, N. Homer, G. Marth, G. Abecasis, R. Durbin; 1000 Genome Project Data Processing Subgroup, The Sequence Alignment/Map format and SAMtools. Bioinformatics 25, 2078-2079 (2009). doi:10.1093/bioinformatics/btp352 Medline

37. S. Anders, P. T. Pyl, W. Huber, HTSeq-A Python framework to work with high- throughput sequencing data. Bioinformatics 31, 166-169 (2015). doi:10.1093/bioinformatics/btu638 Medline

38. A. Yates, W. Akanni, M. R. Amode, D. Barrell, K. Billis, D. Carvalho-Silva, C. Cummins, P. Clapham, S. Fitzgerald, L. Gil, C. G. Girón, L. Gordon, T. Hourlier, S. E. Hunt, S. H. Janacek, N. Johnson, T. Juettemann, S. Keenan, I. Lavidas, F. J. Martin, T. Maurel, W. McLaren, D. N. Murphy, R. Nag, M. Nuhn, A. Parker, M. Patricio, M. Pignatelli, M. Rahtz, H. S. Riat, D. Sheppard, K. Taylor, A. Thormann, A. Vullo, S. P. Wilder, A. Zadissa, E. Birney, J. Harrow, M. Muffato, E. Perry, M. Ruffier, G. Spudich, S. J. Trevanion, F. Cunningham, B. L. Aken, D. R. Zerbino, P. Flicek, Ensembl 2016. Nucleic Acids Res. 44, D710-D716 (2016). doi:10.1093/nar/gkv1157 Medline

39. A. T. L. Lun, K. Bach, J. C. Marioni, Pooling across cells to normalize single-cell RNA sequencing data with many zero counts. Genome Biol. 17, 75 (2016). doi:10.1186/s13059-016-0947-7 Medline

40. A. T. L. Lun, D. J. McCarthy, J. C. Marioni, A step-by-step workflow for low-level analysis of single-cell RNA-seq data with Bioconductor. F1000Res. 5, 2122 (2016). doi:10.12688/f1000research.9501.2 Medline

41. P. Brennecke, S. Anders, J. K. Kim, A. A. Kołodziejczyk, X. Zhang, V. Proserpio, B. Baying, V. Benes, S. A. Teichmann, J. C. Marioni, M. G. Heisler, Accounting for technical noise in single-cell RNA-seq experiments. Nat. Methods 10, 1093-1095 (2013). doi:10.1038/nmeth.2645 Medline

42. L. Haghverdi, A. T. L. Lun, M. D. Morgan, J. C. Marioni, Batch effects in single-cell RNA-sequencing data are corrected by matching mutual nearest neighbors. Nat. Biotechnol. 36, 421-427 (2018). doi:10.1038/nbt.4091 Medline

43. L. Mclnnes, J. Healy, N. Saul, L. Großberger, UMAP: Uniform Manifold Approximation and Projection. J. Open Source Softw. 3, 861 (2018). doi:10.21105/joss.00861

44. D. J. McCarthy, K. R. Campbell, A. T. L. Lun, Q. F. Wills, Scater: Pre-processing, quality control, normalization and visualization of single-cell RNA-seq data in R. Bioinformatics 33, 1179-1186 (2017). doi:10.1093/bioinformatics/btw777 Medline

45. P. Langfelder, B. Zhang, S. Horvath, Defining clusters from a hierarchical cluster tree: The Dynamic Tree Cut package for R. Bioinformatics 24, 719-720 (2008). doi:10.1093/bioinformatics/btm563 Medline

46. Z. Gu, R. Eils, M. Schlesner, Complex heatmaps reveal patterns and correlations in multidimensional genomic data. Bioinformatics 32, 2847-2849 (2016). doi:10.1093/bioinformatics/btw313 Medline

47. A. Scialdone, K. N. Natarajan, L. R. Saraiva, V. Proserpio, S. A. Teichmann, O. Stegle, J. C. Marioni, F. Buettner, Computational assignment of cell-cycle stage from single-cell transcriptome data. Methods 85, 54-61 (2015). doi:10.1016/i.ymeth.2015.06.021 Medline

48. A. Liaw, M. Wiener, Classification and regression by randomForest. $R$ News 2,18 22 (2002).http://dx.doi.org/10.1057/9780230509993

49. L. Haghverdi, F. Buettner, F. J. Theis, Diffusion maps for high-dimensional singlecell analysis of differentiation data. Bioinformatics 31, 2989-2998 (2015) doi:10.1093/bioinformatics/btv325 Medline

50. P. Angerer, L. Haghverdi, M. Büttner, F. J. Theis, C. Marr, F. Buettner, destiny: Diffusion maps for large-scale single-cell data in R. Bioinformatics 32, 1241-1243 (2016). doi:10.1093/bioinformatics/btv715 Medline

51. X. Ibarra-Soria, W. Jawaid, B. Pijuan-Sala, V. Ladopoulos, A. Scialdone, D. J. Jörg, R. C. V. Tyser, F. J. Calero-Nieto, C. Mulas, J. Nichols, L. Vallier, S. Srinivas, B. D. Simons, B. Göttgens, J. C. Marioni, Defining murine organogenesis at single-cell resolution reveals a role for the leukotriene pathway in regulating blood progenitor formation. Nat. Cell Biol. 20, 127-134 (2018). doi:10.1038/s41556-017-0013-z Medline

52. H. M. T. Choi, M. Schwarzkopf, M. E. Fornace, A. Acharya, G. Artavanis, J. Stegmaier, A. Cunha, N. A. Pierce, Third-generation in situ hybridization chain reaction: Multiplexed, quantitative, sensitive, versatile, robust. Development 145 , dev165753 (2018). doi:10.1242/dev.165753 Medline

53. B. Gu, E. Posfai, J. Rossant, Efficient generation of targeted large insertions by microinjection into two-cell-stage mouse embryos. Nat. Biotechnol. 36, 632-637 (2018). doi:10.1038/nbt.4166 Medline 


\section{ACKNOWLEDGMENTS}

We thank S. Meilhac, M. Mommersteeg, P. Riley, R. Sambasivan and M. Torres for valuable discussions and comments. We acknowledge the Single Cell Genomics Core Facility at the Wellcome Sanger Institute for processing SMART-seq2 libraries. S.S. is a member of the British Heart Foundation (BHF) Oxford Centre of Regenerative Medicine and BHF Oxford Centre of Research Excellence. DNA encoding tdTomato was kindly provided by R. Y. Tsien (HHMI/UCSD). We thank N. Ashley at the MRC Weatherall Institute of Molecular Medicine single cell facility for his advice on single cell workflows and the Micron Advanced Bioimaging Unit (supported by Wellcome Awards 091911/B/10/Z and 107457/Z/15/Z and BBSRC ALERT13 Award BB/L014750/1). X.I.-S. is currently affiliated with Computational Biology, Functional Genomics, GSK Medicines Research Centre, Gunnels Wood Road, Stevenage, SG1 2NY, UK. Funding: This work was funded by Wellcome Awards 108438/Z/15/Z and 105031/C/14/Z (S.S. and J.C.M.), Wellcome Senior Investigator Award 103788/Z/14/Z (S.S.), BHF Immediate Fellowship FS/18/24/33424 (R.C.V.T.) and core funding from the European Molecular Biology Laboratory and Cancer Research UK (J.C.M.) and the Howard Hughes Medical Institute (P.J.K.). Author contributions:

R.C.V.T., X.I.-S., J.C.M. and S.S. conceived of the study, interpreted the data and wrote the manuscript. R.C.V.T. conducted all whole mount immuno-

histochemistry, in situ hybridization experiments, imaging and lineage analysis. X.I.-S. performed all computational analyses. R.C.V.T., S.S. and A.M.A.M. dissected and processed embryos for single-cell library preparation. K.M. and P.J.K. performed all lightsheet experiments. T.A.H.v.d.B. and A.S. performed initial scRNA-seq data analyses. SJ and JG generated the Mab2112-iCreERT2 transgenic line. P.J.K., J.C.M. and S.S. supervised the study. All authors read and approved the final manuscript. Authors declare no competing interests. Data and materials availability: The Mab2/12-CreERT2 mouse line is available from S.S. under a material transfer agreement with the University of Oxford. Raw sequencing data has been deposited in the European Nucleotide Archive database under study PRJEB14363 (sample accession identifiers provided in Extended Data 3), and ArrayExpress under accession E-MTAB-7403. The raw count matrices for the unbiased and reference datasets are provided in data S1 and $\mathrm{S} 2$, respectively. Associated sample metadata are available in extended data S3 and S4, respectively. Raw and processed data are also available as R data objects from

https:/content.cruk.cam.ac.uk/imlab/mouseEmbryonicHeartAtlas/. All code used for data analysis, figure generation and the shiny app is available at https:/github.com/MarioniLab/mouseHeart2020.

\section{SUPPLEMENTARY MATERIALS}

science.sciencemag.org/cgi/content/full/science.abb2986/DC1

Materials and Methods

Supplementary Text

Figs. S1 to S20

Tables S1 to S5

References (34-53)

MDAR Reproducibility Checklist

Movies S1 to S14

Data S1 to S4

13 February 2020; resubmitted 13 August 2020

Accepted 17 December 2020

Published online 7 January 2021

10.1126/science.abb2986 
A

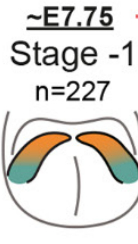

$\square$ FHF $\square$ SHF $\square$ CM

C

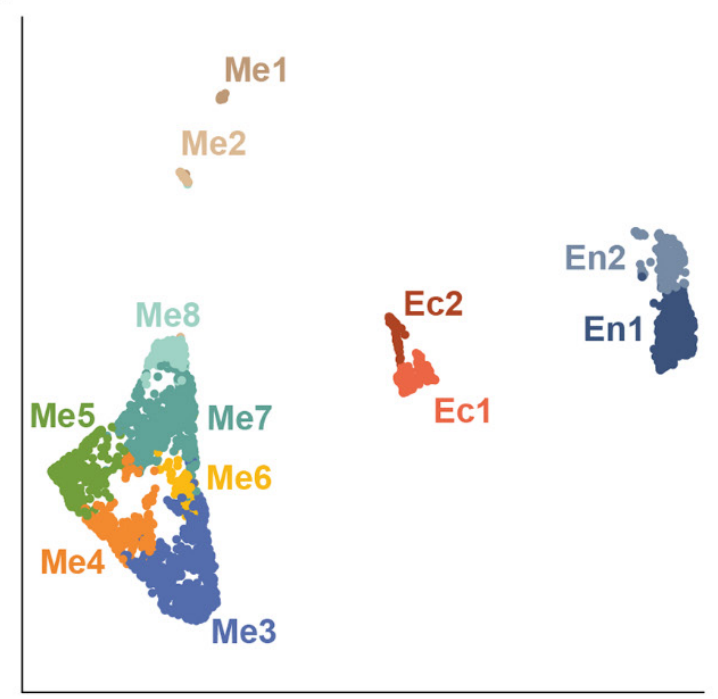

B

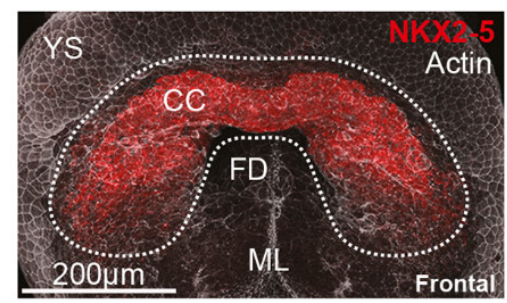

E

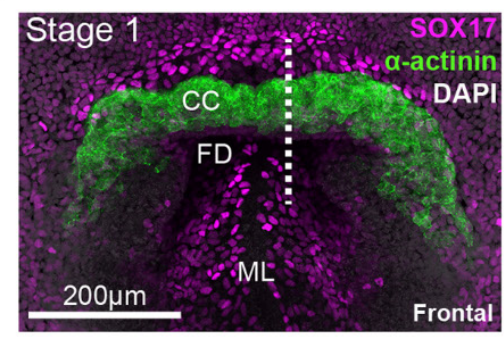

G

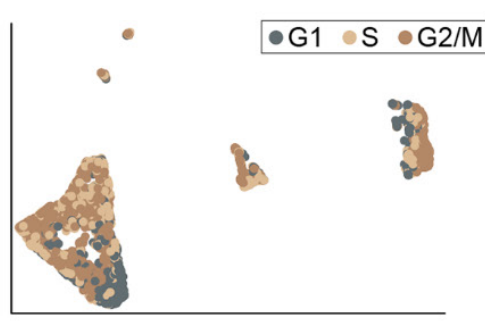

$\mathbf{F}$

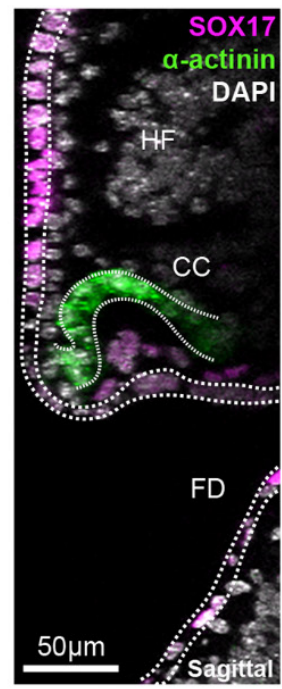

D
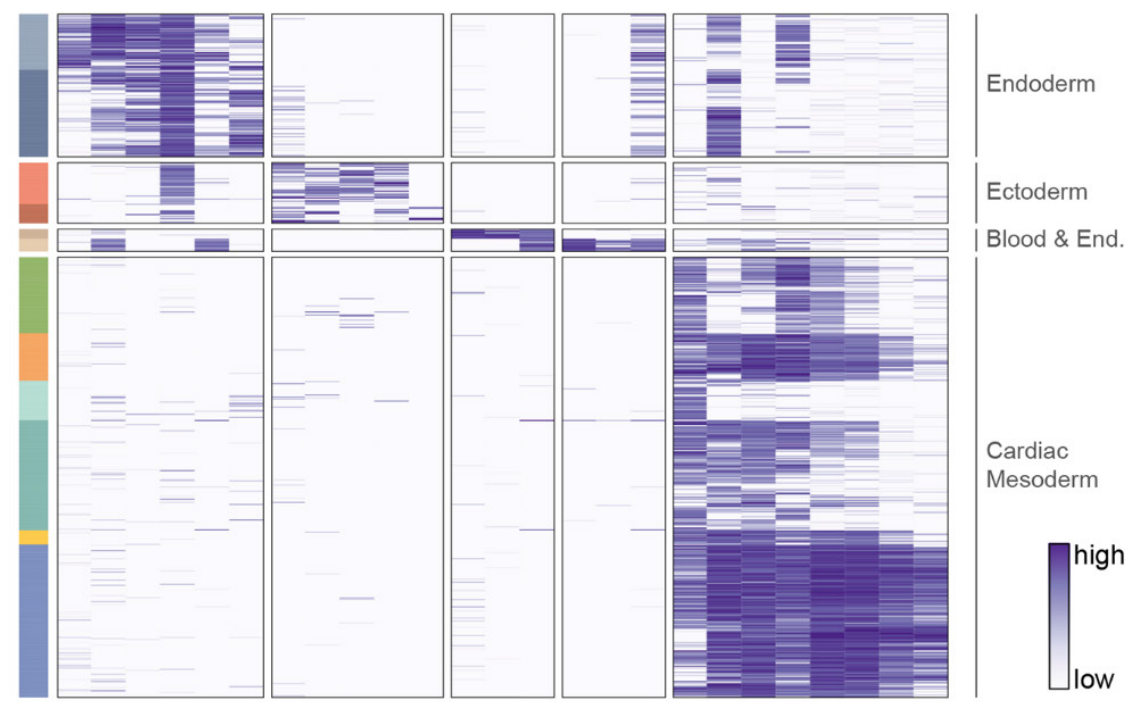

H

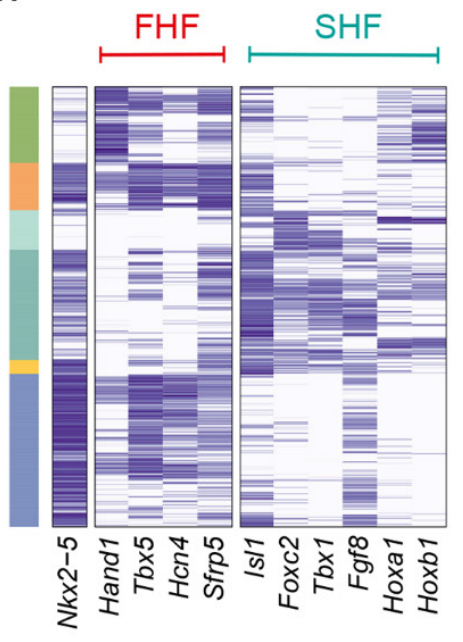

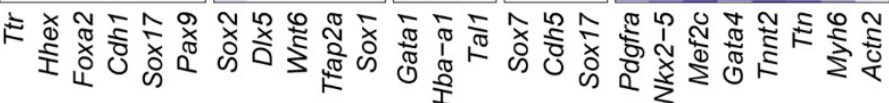


Fig. 1. Single-cell resolution spatiotemporal analysis of the forming murine heart. (A) Schematic of crescent and linear heart tube stages collected for scRNA-seq. $n=$ number of single cells that passed quality control. FHF, first heart field; SHF, second heart field; CM, cardiomyocytes. (B) Maximum intensity projection (MIP) of a stage 1 cardiac crescent region, marked by NKX2-5 protein expression (movie S1). The dotted region highlights the area microdissected for collecting cells. CC, cardiac crescent; FD, foregut diverticulum; YS, yolk sac; ML, midline. (C) UMAP plot of all cells that passed quality control $(n=3105)$ computed from highly variable genes. Cells with similar transcriptional profiles were clustered into 12 different groups, as indicated by the different colors. (D) Heatmap showing the expression of wellcharacterized marker genes. Cells (rows) are ordered first by cluster and then by hierarchical clustering. Each gene's normalized log expression levels are regularized to be within $[0,1]$. (E) MIP of representative embryo showing SOX17 and sarcomeric $\alpha$-actinin protein (movie S2). This serves as an example of the approach used to anatomically localize in the intact embryo the cell types identified in the scRNA-seq dataset. The dotted line represents the location of the section shown in (F). (F) Sagittal section of the embryo in (E). Dotted lines outline the endoderm (SOX17+) and cardiac crescent ( $\alpha$-actinin +$)$. See also fig. S3. HF, headfold. (G) UMAP plot as in (C) but colored based on the inferred cell cycle stage of each cell. $(H)$ Heatmap of cardiac related clusters showing the expression of genes associated with the first (FHF) and second (SHF) heart fields. 
A

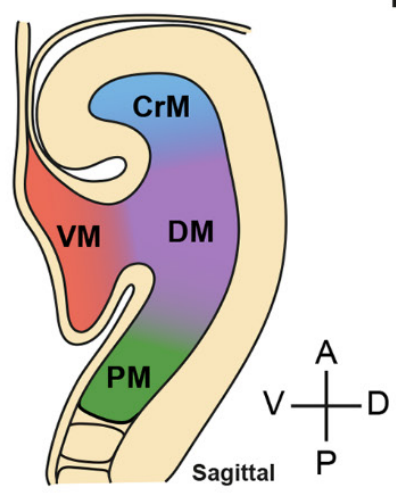

B
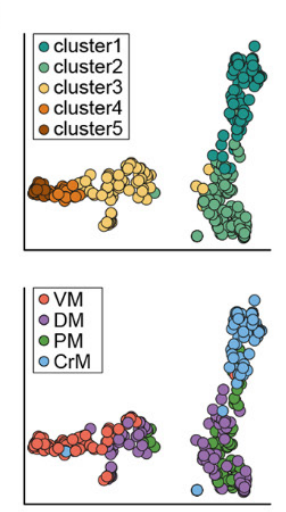

C

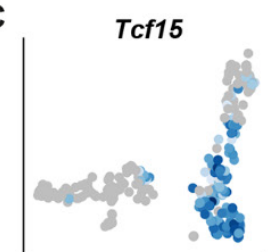

Gata5

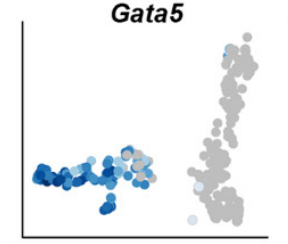

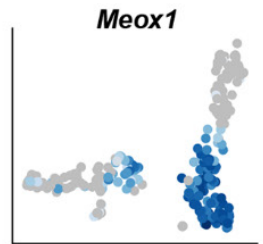

Ttn

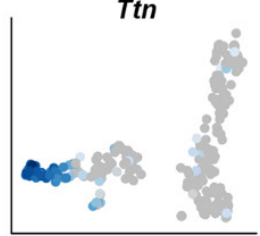

F

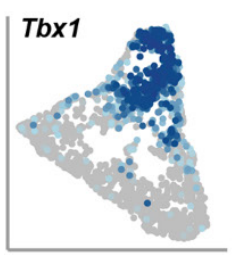

Nkx2-5

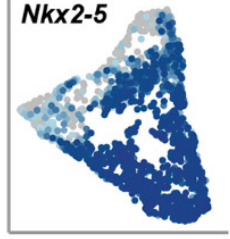

diffusion pseudotime

low

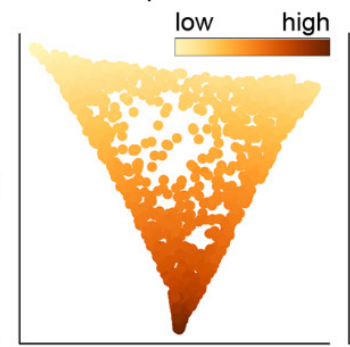

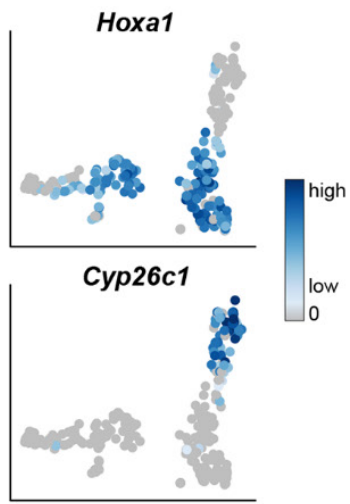

D

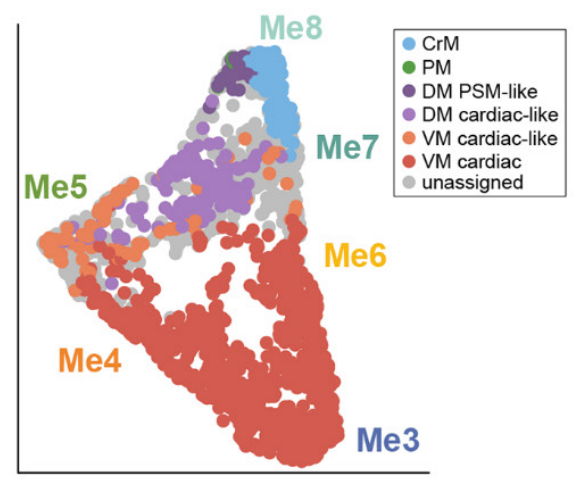

G

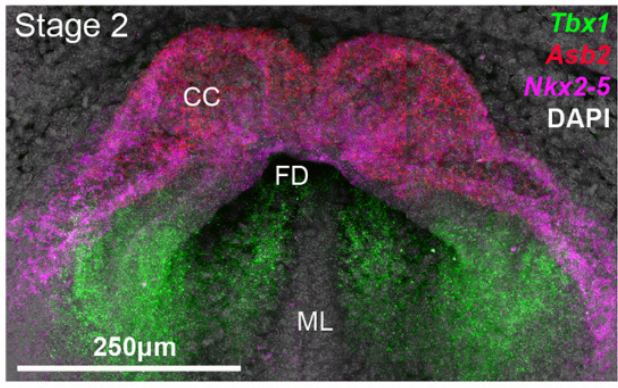

H

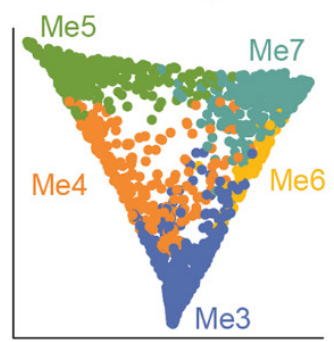

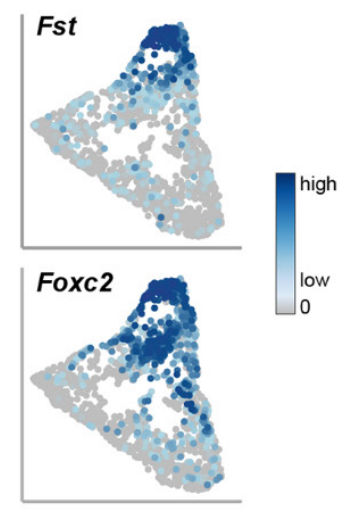

trajectory identification

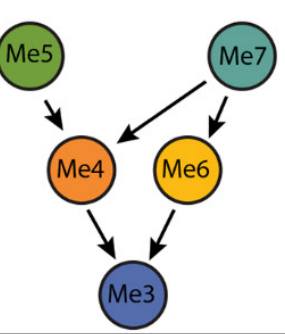

Fig. 2. Classification of cells from clusters Me3-Me8 based on an anatomically resolved mesoderm reference dataset. (A) Schematic indicating the four regions of mesoderm microdissected to generate the reference dataset. Single cells from each region were isolated and used for scRNA-seq. (B) UMAP plot of reference cells that pass quality control, colored by their anatomical origin (top) or clustering based on their transcriptional profiles (bottom). These cells were used to build a random forest classifier. (C) Expression of genes that are important for the random forest, distinguishing the different types of cells. (D) UMAP plot of clusters Me3-Me8 as in Fig. 1C, but colored based on the class assigned by the random forest classifier. Cells in gray cannot be confidently assigned to a single class. (E) Barplot indicating the proportion of cells in each cluster assigned to the different classes. Numbers indicate the number of cells. (F) UMAP plots showing the expression of genes used to identify clusters Me7 and Me8. (G) Maximum intensity projection of a stage 2 crescent showing expression of Nkx2-5 and Tbx1 using hybridization chain reaction (movie S3). (H) Diffusion map of cells from the cardiac mesoderm meta-cluster (Me3-Me7, $n=1772$ ); the first two diffusion components are shown. On the left, cells are colored by their cluster annotation as in Fig. 1C. In the middle, the color gradient indicates diffusion pseudotime measurements for each cell. On the right, schematic diagram summarizing the trajectories identified, linking the progenitor clusters Me5 and Me7 to the mature cardiomyocytes of Me3 (see also fig. S9). Color scales in (C) and (F) represent $\log _{2}$ normalized counts. 
A
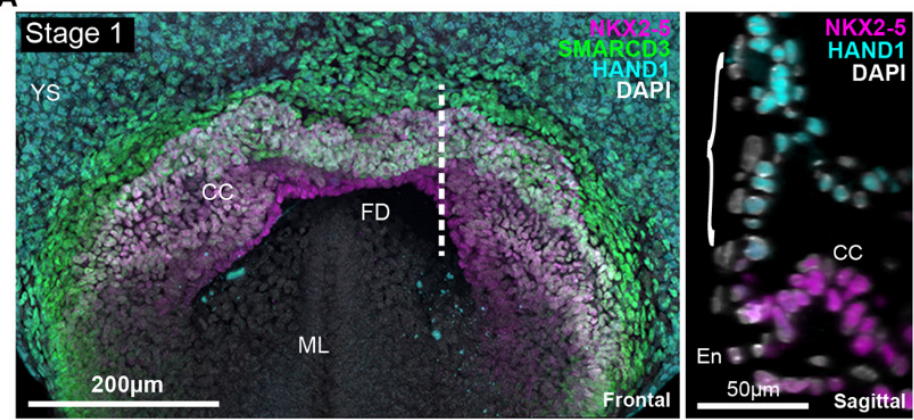

B

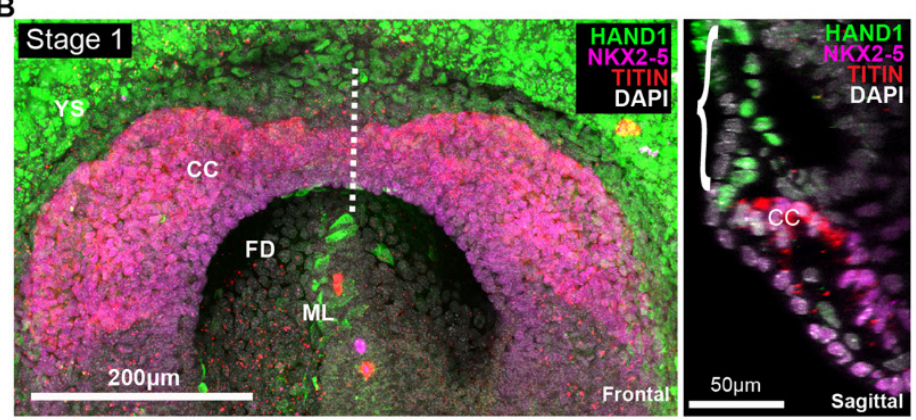

C
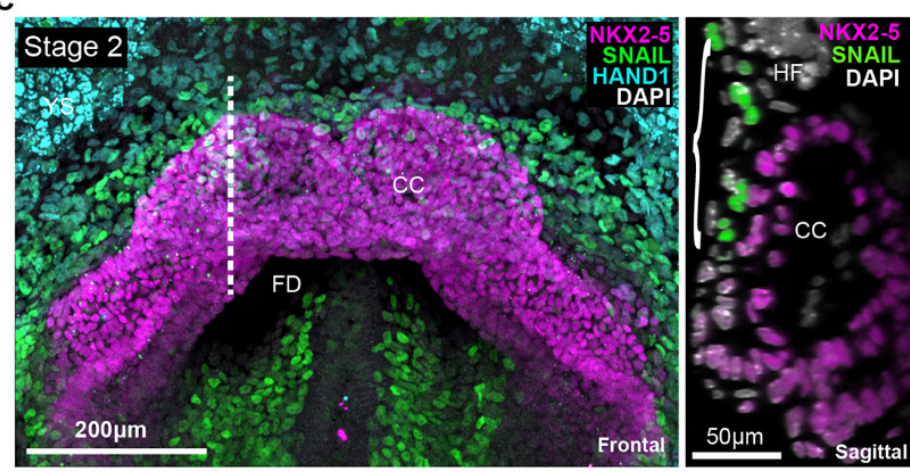

D
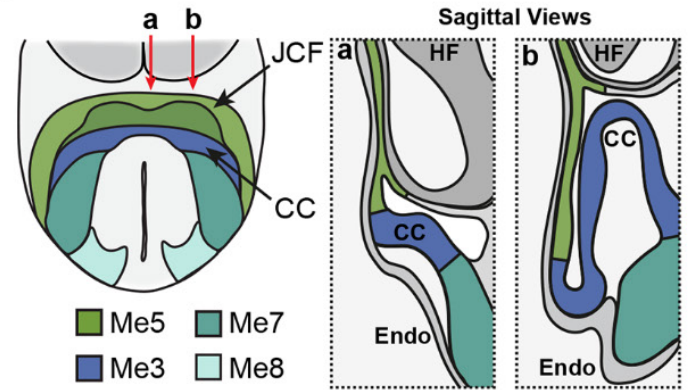

E

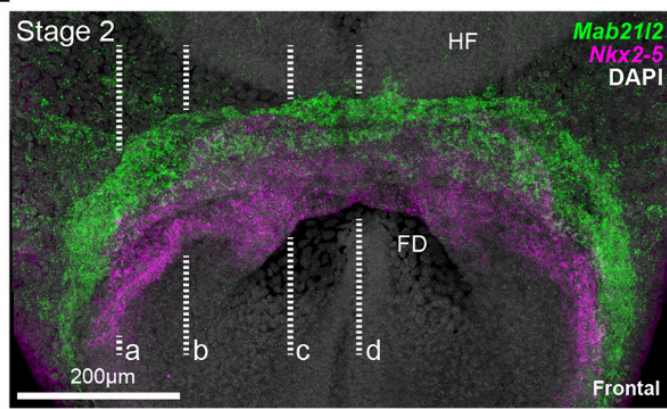

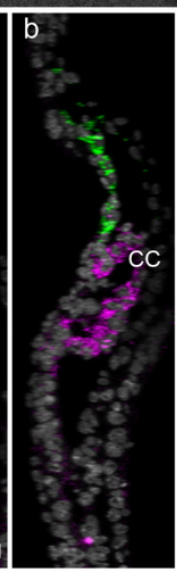

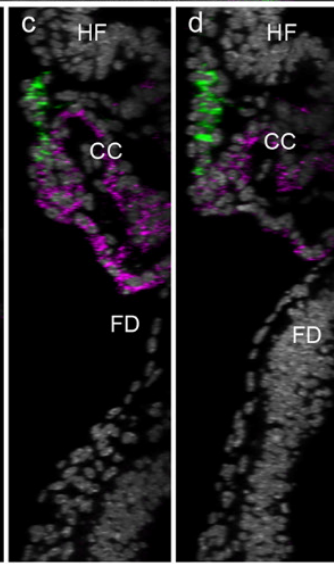

$\mathbf{F}$
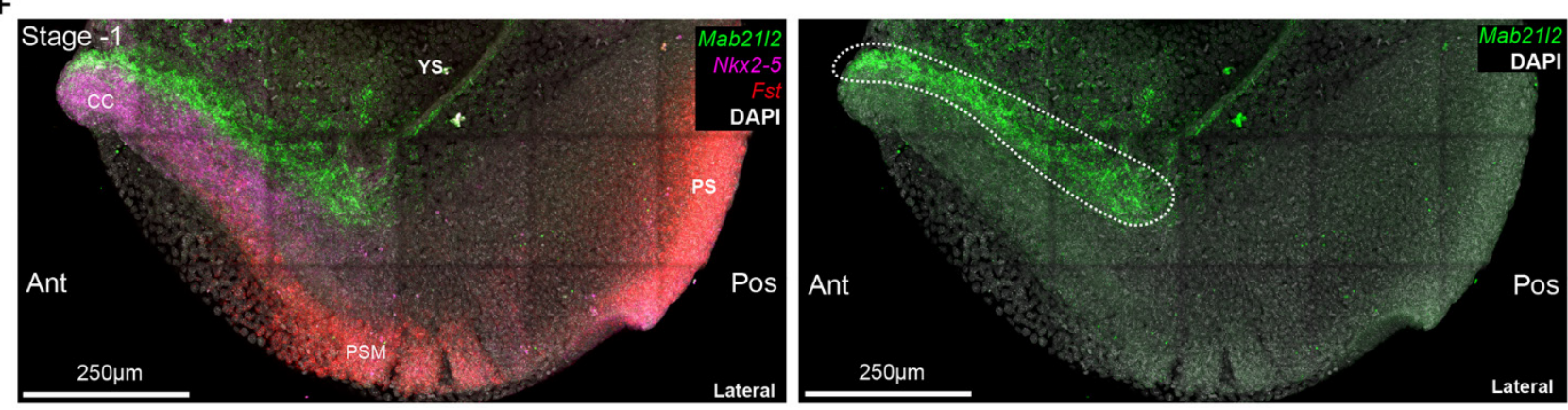
Fig. 3. Identification of a transcriptionally and anatomically distinct cell type with cardiac progenitor like properties. (A to C) Maximum intensity projections (MIP) of immunostained embryos revealing anatomical localization of Me5 (brackets). Dotted line indicates the location of the sagittal section shown at right in each panel. YS, yolk sac; CC, cardiac crescent; FD, foregut diverticulum; ML, midline; En, endoderm; HF, headfold. (D) Schematic highlighting the localization of Me5 [juxta-cardiac field (JCF)] and $\mathrm{Me} 7 / 8$, relative to the myocardium of the cardiac crescent (Me3). (E) MIP of hybridization chain reaction (HCR) staining of an embryo at stage 2 revealing the distinct anatomical localization of JCF cells marked by Mab21/2 (movie S6). Dotted lines (a-d) correspond to sagittal sections shown below. (F) Left, MIP of lateral view of HCR on a stage -1 embryo showing distinct expression domains of Mab2112, Nkx2-5 and Fst (movie S7). Ant, anterior; Pos, posterior; PS, primitive streak; YS, yolk sac; PM, paraxial mesoderm. Right panel shows only Mab21/2 expression highlighting location of Me5 cells (white dotted region). 
A
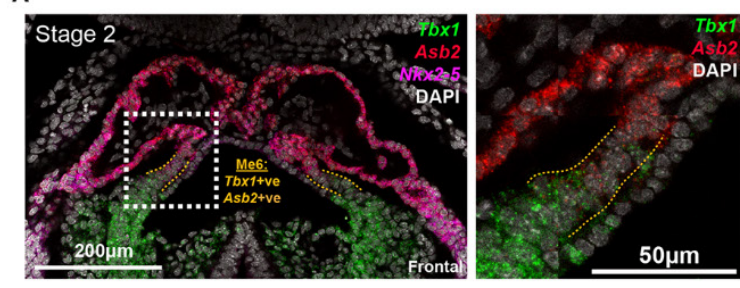

D
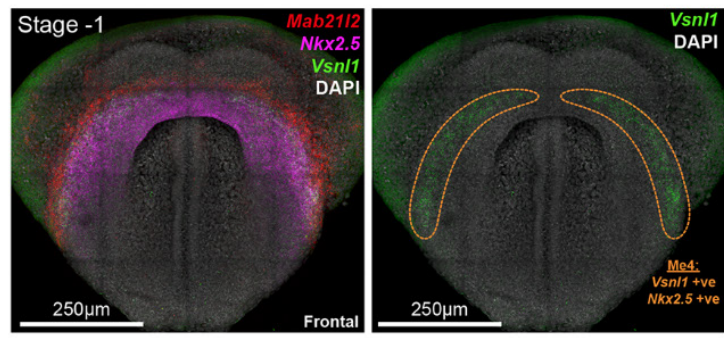

E

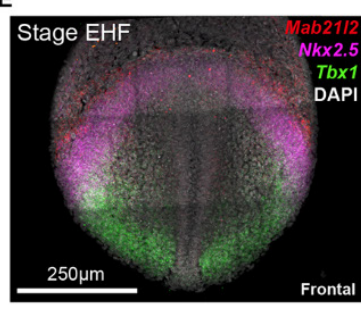

$\mathbf{F}$

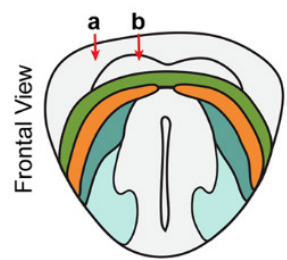

Stage -1

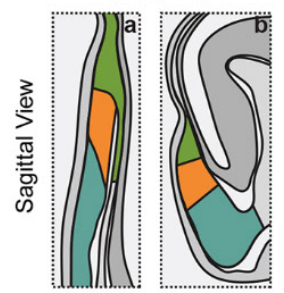

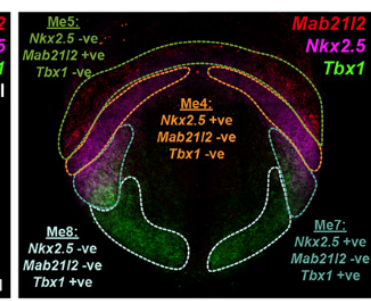

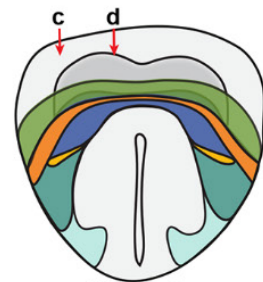

Stage 1

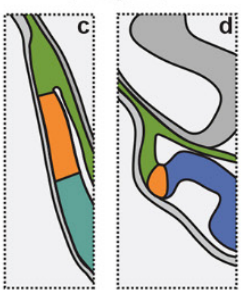

B
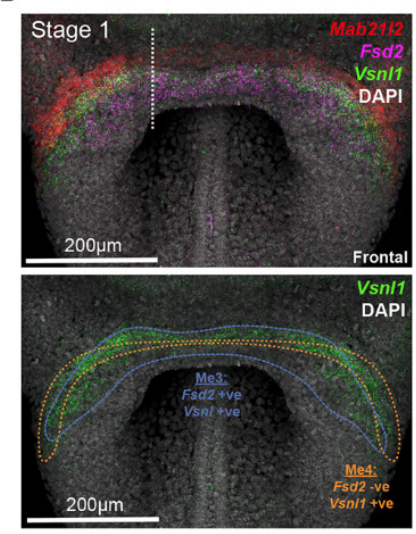

C

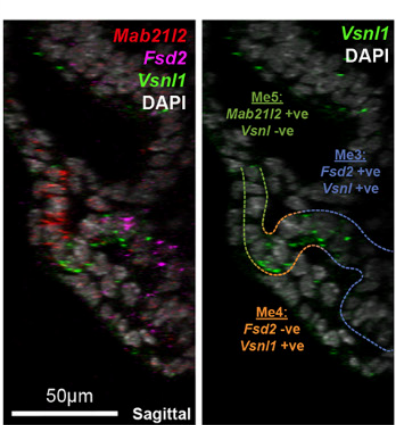

e f $g$
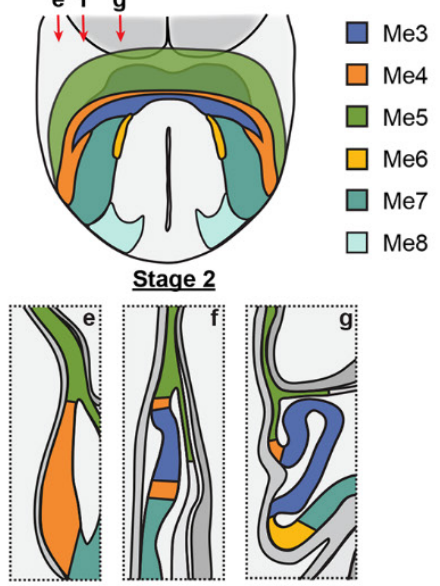

Fig. 4. Anatomical identification of transition states during cardiomyocyte differentiation. (A) Coronal section of a hybridization chain reaction (HCR) stained stage 2 embryo, showing the anatomical location of Me6 (dotted orange lines) marked by coexpression of Tbx1 and Asb2. Dotted white box represents region of higher magnification at right. (B) Maximum intensity projection (MIP) of HCR on stage 1 embryo showing expression of Mab21/2, Vsnl1 and Fsd2 to identify cluster Me4. Colored dotted lines correspond to Me3 and Me4 (movie S8). White dotted line shows location of section in (C). (C) Sagittal section of embryo in (B) highlighting locations of Me3, Me4 and Me5. (D) MIP of HCR on stage -1 embryo showing Mab21l2, Vsnl1 and Nkx2-5, to identify cluster Me4 (yellow dotted line) (movie S11). (E) MIP of an early headfold (EHF) stage embryo, prior to crescent formation, showing expression of Mab21/2, Tbx1 and Nkx2-5, to identify clusters Me4, Me5, Me7 and Me8. Colored dotted lines outline corresponding regions. (F) Schematic highlighting the anatomical location of all mesoderm transcriptional states identified at multiple stages of heart formation. Red arrows represent the location of sagittal sectional views. 

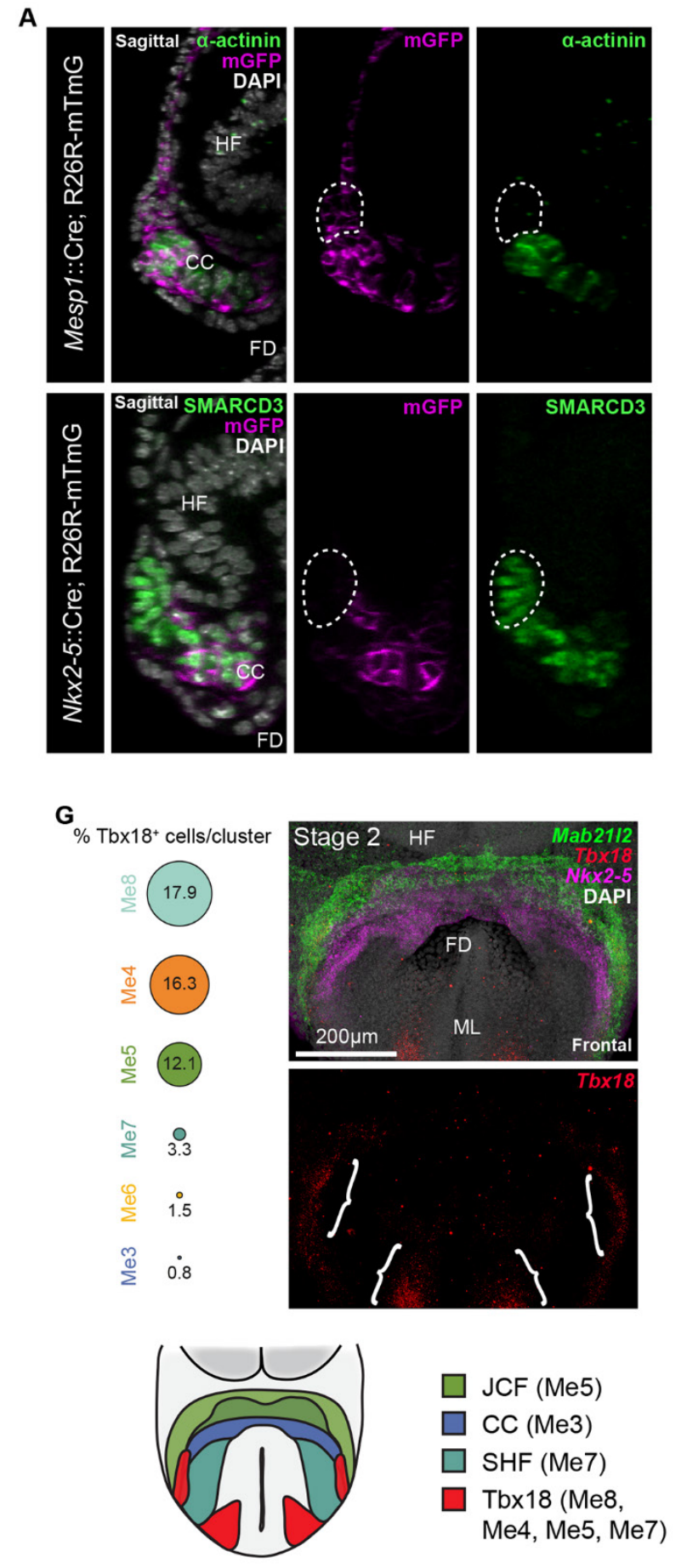

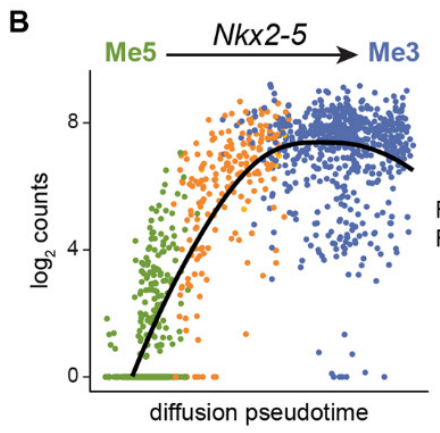

C

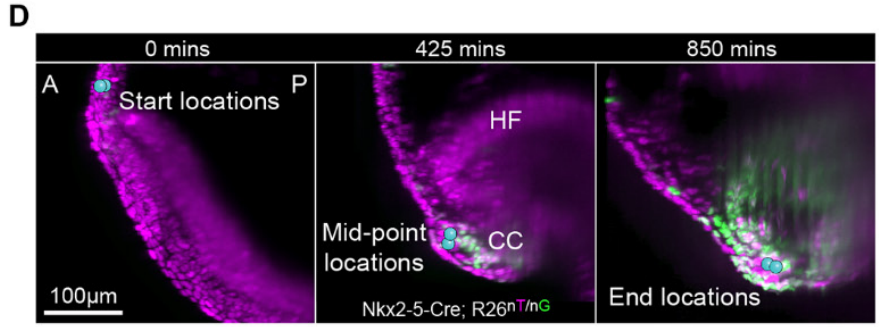

E
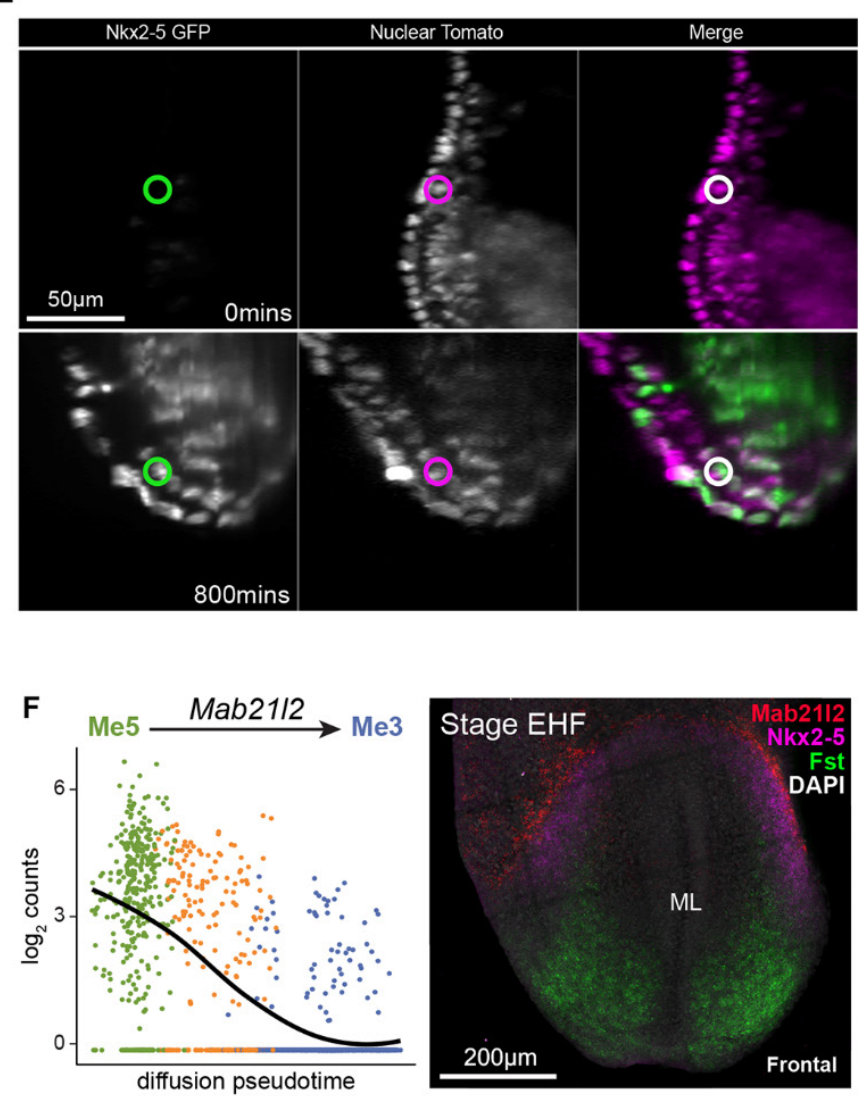
Fig. 5. The JCF represents a cardiac progenitor population. (A) Top, sagittal section of Mesp1-Cre; R26R$\mathrm{mTmG}$ embryos immunostained for $\alpha$-actinin and GFP $(n=6)$. Bottom, section of Nkx2-5-Cre; R26R-mTmG embryos immunostained for SMARCD3 and GFP $(n=4)$. Dotted regions highlight the JCF, revealing it is derived from Mesp1, but not Nkx2-5, expressing cells. HF; headfold; FD, foregut diverticulum; CC, cardiac crescent. (B) Nkx2-5 expression levels plotted against the diffusion pseudotime of all cells consistent with the trajectory from Me5 to Me3. Points are colored based on their assigned cluster. Nkx2-5 is up-regulated during the transition from JCF toward cardiomyocytes. The line is the quadratic local linear fit of the expression levels as a function of pseudotime. (C) Schematic diagrams highlighting the relative positions of Me3-Me5 during rostral folding and the transition from stage 0 to stage 2 cardiac crescents. (D and E) Frames from time-lapse movie of an E7.5 Nkx2-5-Cre; R26-nTnG embryo imaged over a 16-hour period with light-sheet microscopy. (D) JCF cells migrated from a more rostral position toward the developing crescent (blue spheres $=$ representative tracked cells). A, anterior; P, posterior. (E) Nkx2-5 was up-regulated by JCF cells, as shown by an increase in the nuclear expression of GFP. Circles indicate the same cell tracked across time, shown in different channels. Representative cells also shown in movies S12 and S13. (F) On the left, Mab21/2 gene expression levels are plotted against the diffusion pseudotime of all cells consistent with the trajectory from Me5 to Me3. Points are colored based on their assigned cluster. Mab21/2 expression is downregulated during the transition from the JCF toward cardiomyocytes. At right, maximum intensity projection (MIP) of HCR on an early headfold stage (EHF) embryo, prior to crescent formation, revealing the early rostral expression of Mab2112 in JCF progenitors proximal to Nkx2-5 and Fst expression. 3D rendering of expression is shown in movie S14. ML, midline. (G) Tbx18 is expressed in multiple cell clusters. The percentages of Tbx18-positive cells from each cluster are shown as proportionally sized circles. At right, MIP of HCR for Tbx18, Mab21/2 and Nkx2-5 in a stage 2 embryo, highlighting location of Tbx18 expression (brackets) (movie S6). Schematic at bottom, highlights Tbx18 expression domains in the anterior crescent region of the developing embryo. 
A

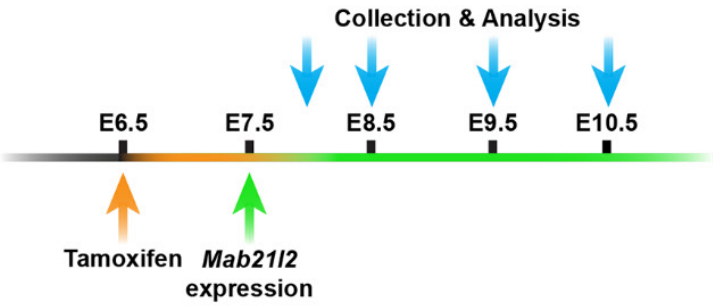

B

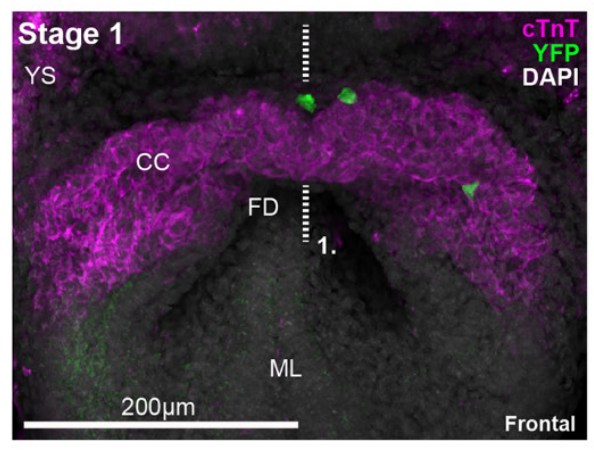

C
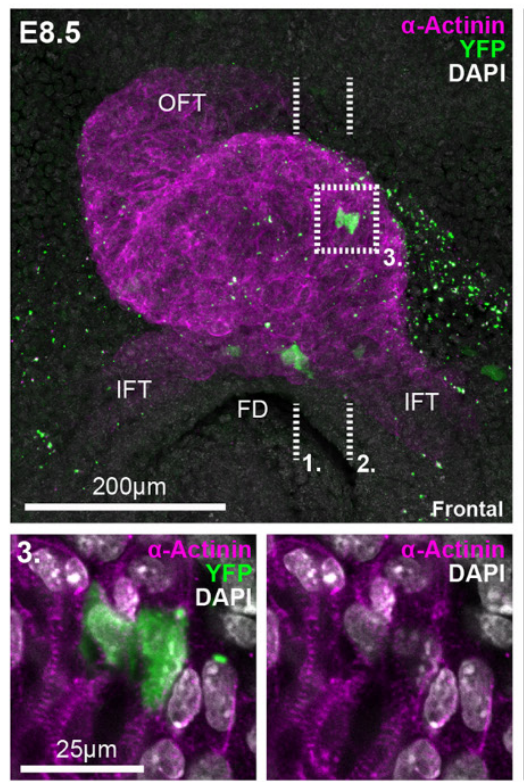

E
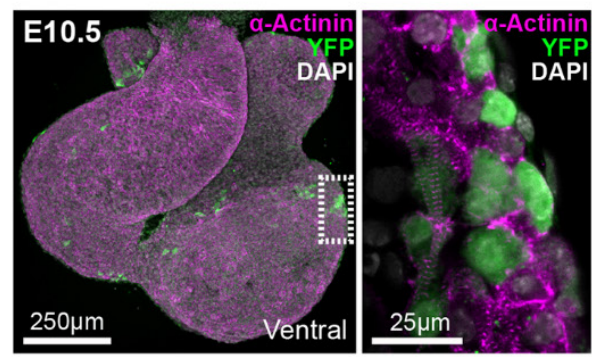
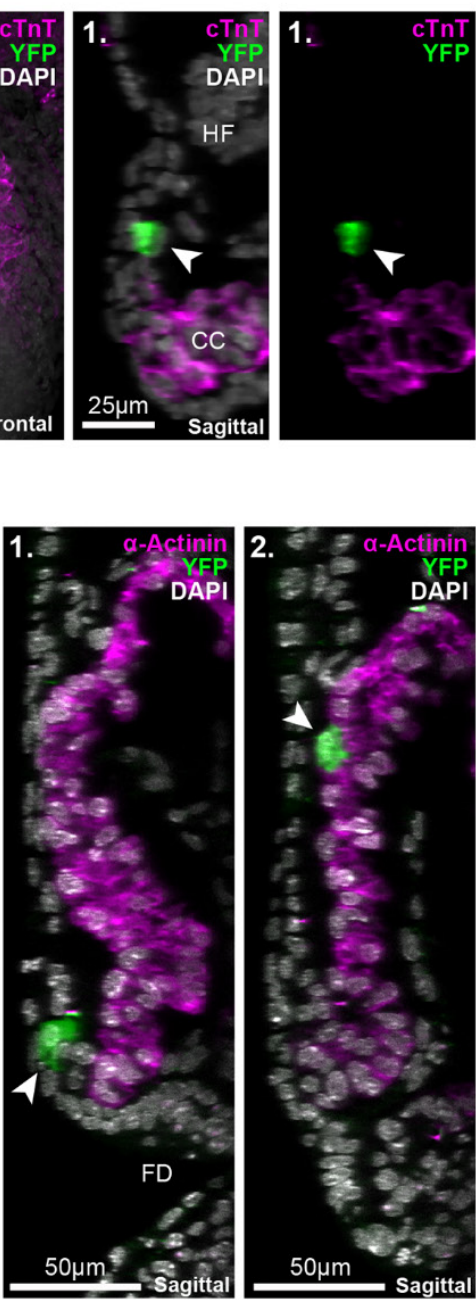

$\mathrm{F}$

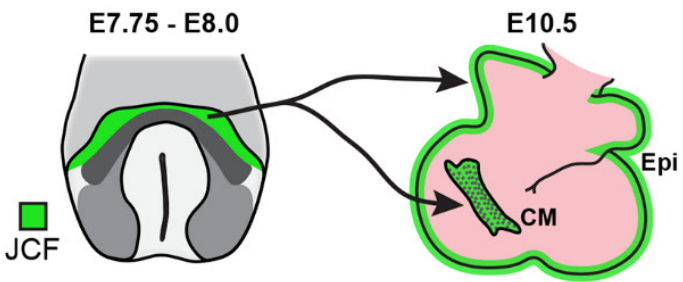

D
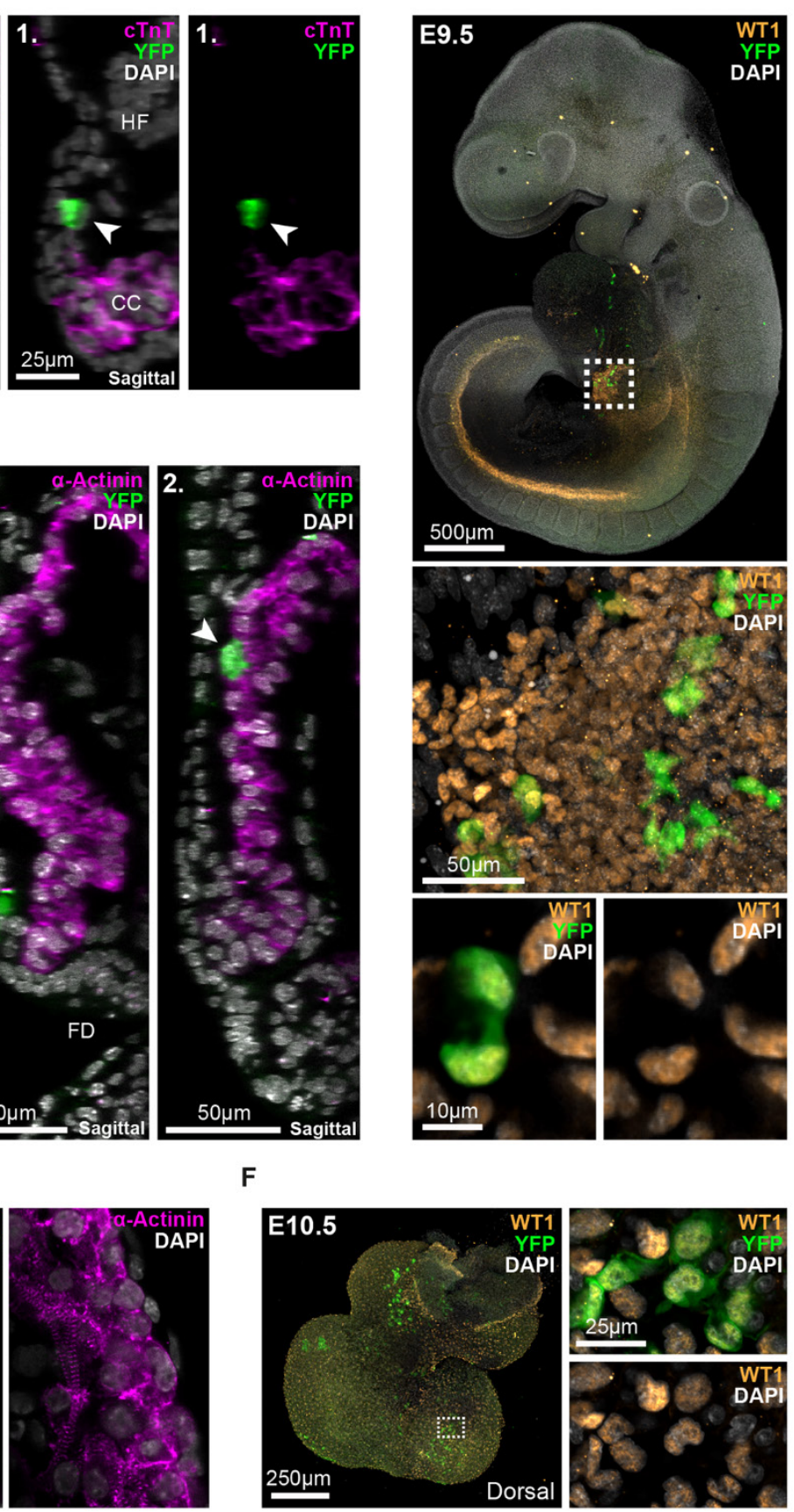
Fig. 6. Juxta-cardiac field progenitors contribute to cardiomyocytes and epicardium. (A) Schematic showing the experimental design of lineage labeling experiments using a Mab21/2iCreERT2 transgenic mouse line. At right, a schematic highlighting that labeling of the juxtacardiac field (JCF) between E7.75 and E8.0 leads to labeling of both cardiomyocytes (CM) and the epicardium (Epi) at E10.5. (B) Maximum intensity projection (MIP) of a stage 1 Mab2112-iCreERT2; R26R-YFP embryo immunostained for cardiac troponin T (cTnT) and YFP. The dotted line represents the location of the sagittal section at right. YFP positive cells (arrows) are located in the JCF and do not express cardiomyocyte marker CTnT. CC, cardiac crescent; FD, foregut diverticulum; YS, yolk sac; ML, midline; HF, headfold. (C) MIP of a looping heart tube from an E8.5 Mab21I2-iCreERT2; R26R-YFP embryo immunostained for $\alpha$-actinin and YFP. Dotted lines show locations of sagittal sections at right. Arrows highlight the location of recombined YFP cells within the myocardium (2.) and maintained within the JCF progenitor region (1.). The dotted box (3.) shows zoomed in view of JCF derived cardiomyocytes with sarcomeric striations of $\alpha$-actinin. OFT, outflow tract; IFT, inflow tract. (D) MIP of an E9.5 embryo immunostained for WT1 and YFP. WT1 marks both the nephrogenic cord and proepicardium (boxed). Below are high magnification views of the proepicardium highlighting the coexpression of YFP and WT1. (E and F) Immunostaining of E10.5 hearts showing coexpression of $\alpha$-actinin (E) and WT1 (F) with YFP positive cells, highlighting the dual potential of the JCF. Dotted boxes represent zoomed in regions. Dorsal and ventral views shown. 